\title{
Detection of fluorescent organic nanoparticles by confocal laser endomicroscopy in a rat model of Barrett's esophageal adenocarcinoma
}

This article was published in the following Dove Press journal:

International Journal of Nanomedicine

30 October 2015

Number of times this article has been viewed

\author{
Elisa Dassie, ${ }^{1,2, *}$ Diletta \\ Arcidiacono, 2,3,* Iga \\ Wasiak, ${ }^{4}$ Nunzio Damiano, ${ }^{5}$ \\ Luigi Dall'Olmo, ${ }^{6}$ \\ Cinzia Giacometti, ${ }^{7}$ \\ Sonia Facchin, ${ }^{3}$ Mauro \\ Cassaro, ${ }^{7}$ Ennio Guido, ${ }^{8}$ \\ Franca De Lazzari, 8 Oriano \\ Marin,, 90 Tomasz Ciach, ${ }^{4}$ \\ Suzanne Fery-Forgues, 1,,12 \\ Alfredo Alberti, 1,2 \\ Giorgio Battaglia, ${ }^{13}$ \\ Stefano Realdon ${ }^{13}$ \\ 'Department of Molecular Medicine, \\ University of Padua, ${ }^{2}$ Venetian Institute of \\ Molecular Medicine, ${ }^{3}$ Department of Surgery, \\ Oncology and Gastroenterology, University \\ of Padua, Padua, Italy; ${ }^{4}$ Faculty of Chemical \\ and Process Engineering, Warsaw University \\ of Technology, Warsaw, Poland; ${ }^{5}$ Department \\ of Biomedical Sciences, University of \\ Padua, Padua, ' ${ }^{6}$ epartment of Emergency \\ Medicine, "Santi Giovanni e Paolo" Hospital, \\ Venice, ${ }^{7}$ Anatomic Pathology Unit, ULSS \\ 15, Alta Padovana, Camposampiero, \\ ${ }^{8}$ Gastroenterology Unit, Sant'Antonio \\ Hospital, 'Interdepartmental Research \\ Centre for Innovative Biotechnologies \\ (CRIBI), University of Padua, ${ }^{10}$ Proteomics \\ Facility, Azienda Ospedaliera di Padova, \\ Padua, "CNRS, ITAV-USR 3505, Toulouse, \\ France; ${ }^{12}$ Université de Toulouse, ITAV-USR \\ 3505, Toulouse, France; ${ }^{13}$ Endoscopy Unit, \\ Veneto Institute of Oncology (IOV-IRCCS), \\ Padua, Italy \\ *These authors contributed equally \\ to this work
}

Correspondence: Elisa Dassie Department of Molecular Medicine, University of Padua, Via Gabelli 9, 35121 Padua, Italy

Tel +390497923224

Fax +390497923250

Email dassie.elisa@ymail.com
Abstract: For many years, novel strategies for cancer detection and treatment using nanoparticles (NPs) have been developed. Esophageal adenocarcinoma is the sixth leading cause of cancerrelated deaths in Western countries, and despite recent advances in early detection and treatment, its prognosis is still very poor. This study investigated the use of fluorescent organic NPs as potential diagnostic tool in an experimental in vivo model of Barrett's esophageal adenocarcinoma. NPs were made of modified polysaccharides loaded with [4-(dicyanomethylene)-2-methyl-6(4-dimethylaminostyryl)-4H-pyran] (DCM), a well-known fluorescent dye. The NP periphery might or might not be decorated with ASYNYDA peptide that has an affinity for esophageal cancer cells. Non-operated and operated rats in which gastroesophageal reflux was surgically induced received both types of NPs (NP-DCM and NP-DCM-ASYNYDA) by intravenous route. Localization of mucosal NPs was assessed in vivo by confocal laser endomicroscopy, a technique which enables a "real time" and in situ visualization of the tissue at a cellular level. After injection of NP-DCM and NP-DCM-ASYNYDA, fluorescence was observed in rats affected by esophageal cancer, whereas no signal was observed in control non-operated rats, or in rats with simple esophagitis or Barrett's esophagus mucosa. Fluorescence was observable in vivo 30 minutes after the administration of NPs. Interestingly, NP-DCM-ASYNYDA induced strong fluorescence intensity 24 hours after administration. These observations suggested that NPs could reach the tumor cells, likely by enhanced permeability and retention effect, and the peptide ASYNYDA gave them high specificity for esophageal cancer cells. Thus, the combination of NP platform and confocal laser endomicroscopy could play an important role for highlighting esophageal cancer conditions. This result supports the potential of this strategy as a targeted carrier for photoactive and bioactive molecules in esophageal cancer diagnosis and treatment. Keywords: confocal laser endomicroscopy, Barrett's esophagus, diagnostics, esophageal adenocarcinoma, fluorescent nanoparticles, heptapeptide

\section{Introduction}

Esophageal adenocarcinoma (EAC) is a major public health concern, particularly in Western countries, due to its high mortality and incidence rising by at least sixfold in the past three decades. ${ }^{1}$ Prognosis is still very poor, with a 5-year survival rate below $20 \%{ }^{2}$ The steps involved in the progression from gastroesophageal reflux disease, its predisposing condition, to Barrett's esophagus (BE), its premalignant disorder, and to cancer, are not yet fully understood. ${ }^{3}$ While BE is found in $5 \%-10 \%$ of patients with chronic gastroesophageal reflux disease, most patients do not progress to EAC. ${ }^{4}$ Moreover, most EAC are diagnosed incidentally and often in advanced stages hardly amenable to cure. ${ }^{5}$ Periodic endoscopic surveillance is the only currently available measure to diagnose early gastric cancer in high-risk populations who have 
pre-cancerous lesions. However, the current surveillance program and procedure of endoscopic diagnosis are labor demanding and very expensive. ${ }^{6}$ Current treatment generally includes either surgery or a combination of radiation and chemotherapy for localized tumors, but only a small portion of patients appears to benefit from it. The optimal strategy for esophageal cancer remains multimodality therapy, which includes radiotherapy, surgery, chemotherapy, and endoscopic therapy. However, all these therapies present some limitations.

Thus, there is an urgent need to identify and validate novel strategies to increase both early diagnosis and drug tropism to cancer sites, thus allowing for the development of more effective treatment. In this context, nanoparticles (NPs) present distinct advantages. They may specifically bind to cancer cells and highlight neoplastic lesions. Their use for targeted cancer treatment is a very active field of research. Indeed, NPs have unique characteristics, they are big enough to avoid rapid clearance by the kidneys. At the same time, they are small enough to navigate easily in the vasculature and cross barriers through small leaky capillaries to reach tumor cells by enhanced permeability and retention effect. ${ }^{7-10}$ NPs offer large surface area, so that surface chemistry allows their physical and biological properties to be widely modified. In particular, they may have prolonged circulation time and additional modification of NPs with various "homing" molecules increases their affinity and specificity for tumor cells.

On the other hand, confocal laser endomicroscopy (CLE) enables in situ and "real time" visualization of the tissues at a cellular level within gastrointestinal tract. ${ }^{11-16}$ The system developed by Mauna Kea Technologies (Paris, France) is based on a Cellvizio ${ }^{\circledR}$ microscopy probe that is passed through the instrument channel of a conventional endoscope. Magnification is approximately $1,000 \times$. Using CLE after topical or intravenous (iv) administration of a fluorescent probe that specifically binds to cancer cells would result in an immediate visualization and quantification of cellular and molecular processes in living tissues.

In the research presented in this paper, we combined the nanomedicine and CLE approaches using NPs as imaging agents. The NPs that were used offered distinct advantages. They were totally organic, and their toxicity was very low. They were made of a modified polysaccharide that provides stability, biocompatibility, and furtivity toward the immune system, and even shows good affinity for cancer cells. ${ }^{17}$ The polysaccharide was loaded with an organic dye, namely 4-(dicyanomethylene)-2-methyl-6-(4-dimethylaminostyryl)4H-pyran (DCM). Finally, the heptapeptide ASYNYDA was covalently grafted at the periphery of the NP as a recognition unit to enhance selectivity (Figure 1). ${ }^{18,19}$ In fact, in the literature, this peptide has been isolated from a phage library, labeled with fluorescein isothiocyanate and topically administered on human esophageal ex-vivo specimens. Increased fluorescence intensity at the site of neoplastic mucosa has been clearly observed, showing that the peptide has high sensitivity and specificity for high-grade dysplasia and EAC in BE. ${ }^{20,21}$ To evaluate the clinical potential of our strategy, we evaluated the ability of NPs both decorated and not decorated with peptides (NP-DCM-ASYNYDA and NP-DCM, respectively) to recognize esophageal tumor cells when administered iv in a rat model of $\mathrm{BE}$ adenocarcinoma. The use of animal models is an important tool for improving the knowledge on both diagnosis and therapy of the disease..$^{22-24}$ The "Kumagai-Hattori" rat model has already been described and validated by previous studies. ${ }^{24-26}$ In the present model, the length of intestinal metaplasia is a compelling evidence that intestinal metaplasia represents a consequence of the exposure of the distal part of rat esophagus to reflux, without any exogenous carcinogen treatment. The phases of EAC development are comparable to what occurs in humans: esophagitis, intestinal metaplasia (the precancerous condition), and EAC. Evidence was given for the value of our NPs for the detection of EAC. The comparison between both types of NPs showed that the presence of peptides was a distinct advantage for selectivity.

\section{Materials and methods}

All chemical reagents were purchased from the indicated suppliers and used as received, without additional purification.

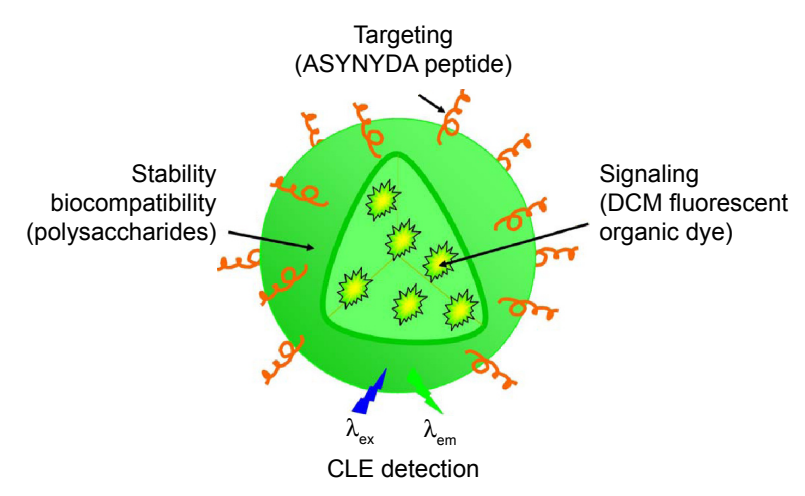

Figure I Schematic representation of the organic fluorescent nanoparticles.

Notes: NPs were made of modified polysaccharides loaded with the fluorescent dye DCM. The NP periphery might or might not be decorated with ASYNYDA peptide that has an affinity for esophageal cancer cells.

Abbreviations: NP, nanoparticle; CLE, confocal laser endomicroscopy; DCM, 4-(dicyanomethylene)-2-methyl-6-(4-dimethylaminostyryl)-4H-pyran; NPs, nanoparticles. 


\section{Synthesis of NPs}

\section{Dextran oxidation}

Dextran (molecular weight $=70 \mathrm{kDa}$, pharmacopeia grade, Pharmacosmos, Holbaek, Denmark) was first oxidized according to a modified protocol of Muangsiri and $\mathrm{Kirsch}^{27}$ and Fuentes et al. ${ }^{28}$ Briefly, $10 \mathrm{~g}$ of dextran was dissolved in $200 \mathrm{~mL}$ of distilled water. Sodium metaperiodate (SigmaAldrich, St Louis, MO, USA) was added in a molar ratio of $1: 10$ (periodate moles/glucose units) to obtain approximately $8 \%$ of glucose ring oxidation. The solution was stirred in the dark at room temperature for 1 hour. Then, the reaction was quenched by the addition of $10 \mathrm{~mL}$ ethylene glycol (Chempur, Piekary Śląskie, Poland). The post-reaction solution was dialyzed against distilled water using a dialysis membrane bag (Carl Roth GmbH + Co. KG, Karlsruhe, Germany, molecular weight cut off $12-14 \mathrm{kDa}$ ) for 3 days, and lyophilized. The final product was stored in the refrigerator in a closed container.

\section{Preparation of fluorescent polysaccharide NPs}

NPs loaded by DCM (Sigma-Aldrich) were made in the microfluidic device manufactured in our laboratory (Figure 2). The device was arranged between two standard microscopy glass slides. Four blunt medical needles (needles for ophthalmic applications, Mifam, Milanowek, Poland) of $0.7 \mathrm{~mm}$ outer diameter and $0.5 \mathrm{~mm}$ inner diameter were inserted into short pieces of well-fitted polytetrafluoroethylene pipes $(1.2 \mathrm{~mm}$ outer diameter, to leave space between naked needles and glass in the mixing region), and arranged as shown in Figure 2. The distance between the input needle A and the output needle end (main mixing zone) was approximately $1 \mathrm{~mm}$. Needles were fixed by polydimethylsiloxane resin. The same resin was also used to form the walls of the flat chamber between glass pieces of approximately $10 \mathrm{~mm}$ inner diameter. The distance between glass slides that determined the height of the chamber was approximately $1.5 \mathrm{~mm}$. The dye was dissolved in absolute ethanol (Chempur) with final concentration $1 \times 10^{-3} \mathrm{M}$. The flow ratio of the dye solution (A) was $1 \mathrm{~mL} / \mathrm{h}$. For the production of dye-loaded polysaccharide NPs, the precipitation solution was prepared using $5 \%$ oxidized dextran and $1 \%$ dodecylamine in distilled water. The precipitation solution was injected through needle $B$ with a flow ratio of $49 \mathrm{~mL} / \mathrm{h}$. The solution flowing out of the microfluidic device was collected and stirred at $30^{\circ} \mathrm{C}$ for 30 minutes. During this time, the formation of fluorescent NPs was observed. To finish the Schiff base reaction, the $\mathrm{pH}$ was slowly increased to $\mathrm{pH} 10$ by addition of $0.5 \mathrm{M}$ sodium hydroxide within 90 minutes, the mixture being continuously stirred and heated. At the end of the reaction, the $\mathrm{pH}$ was adjusted at 7.4 and the obtained solution was dialyzed against distilled water for 30 minutes, using the same membrane as for the dextran oxidation procedure. The prepared dye-loaded polysaccharide NPs were lyophilized with addition of $1 \%$ w/w dextran $70 \mathrm{kDa}$ as a cryoprotectant.

\section{Peptides synthesis}

The synthetic EAC targeting peptides containing the sequence ASYNYDA were prepared by solid phase peptide synthesis using a multiple peptide synthesizer (SyroII, MultiSynTech GmbH, Witten, Germany) on Rink Amide MBHA resin (Novabiochem, Bad Soden, Germany). The fluoren-9ylmethoxycarbonyl (Fmoc) strategy ${ }^{29}$ was used throughout the peptide chain assembly, using $O$-(7-azabenzotriazole1-yl)- $N, N, N^{\prime}, N^{\prime}$-tetramethyluronium hexafluorophosphate ${ }^{30}$ (ChemPep, Wellington, FL, USA) as coupling reagent. The side-chain protected amino acid building blocks were Fmoc-Asp(OtBu)-OH, Fmoc-Asn(Trt)Asn-OH, Fmoc$\mathrm{Ser}(\mathrm{tBu})-\mathrm{OH}$, Fmoc-Lys(Mtt)-OH, and Fmoc-Tyr(tBu)-OH. The acetylation of the $\mathrm{N}$-terminal amino group was performed

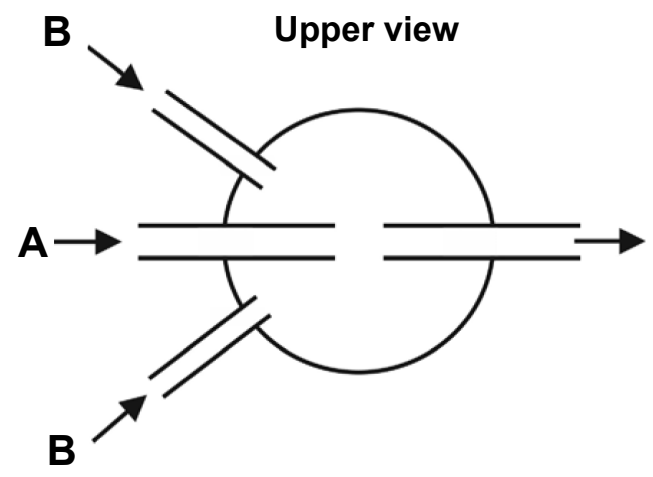

Side view

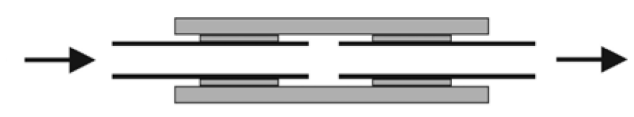

Figure 2 Schematic drawing of microfluidic precipitation device.

Notes: The setup was arranged between two standard microscopy glasses, blunt ophthalmic needles in short polytetrafluoroethylene pipes served as input and output channels. (A) Ethanol solution of organic dye. (B) Precipitating aqueous solution containing oxidized dextran and dodecylamine. Needles were fixed with silicon (polydimethylsiloxane) glue, the same glue was used to form a round hydraulic chamber between glasses. 
by the treatment of the peptidyl-resin with a mixture of 0.015 M HOAt (1-hydroxy-7-azabenzotriazole) (ChemPep), $0.5 \mathrm{M}$ acetic anhydride, $0.125 \mathrm{M} N$-ethyldiisopropylamine in $N, N$-dimethylformamide (Sigma-Aldrich). The Mtt group was selectively removed by treatment with a $10 \%$ acetic acid in dichloromethane followed by a second treatment with $1 \%$ trifluoroacetic acid, 10\% trifluoroethanol in dichloromethane. Cleavage of the peptides was performed by incubating the peptidyl resins with trifluoroacetic acid/ $\mathrm{H}_{2} \mathrm{O} /$ thioanisole/ ethanedithiol/phenol $(10 \mathrm{~mL} / 0.5 \mathrm{~mL} / 0.5 \mathrm{~mL} / 0.25 \mathrm{~mL} / 750 \mathrm{mg})$ for 2.5 hours. Crude peptides were purified by a AKTA ${ }^{\mathrm{TM}}$ basic high-performance liquid chromatography system (GE Healthcare UK Ltd, Little Chalfont, UK) equipped with a Prep Nova-Pak HR C18 (Waters, Milford, MA, USA) reverse phase column high-performance liquid chromatography. Molecular masses of the peptides were confirmed by mass spectrometry (MS) on an ABI 4800 matrix-assisted laser desorption/ionization time-of-flight (MALDI-TOF/TOF) mass spectrometer (Thermo Fisher Scientific, Waltham, MA, USA).

\section{Grafting of the ASYNYDA peptides to fluorescent polysaccharide NPs}

Peptides were grafted on polysaccharide fluorescent NPs as follows. A lyophilized sample of polysaccharide NPs (0.54 g), obtained as described above, was suspended in distilled water, and $1 \mathrm{mg}$ peptide dissolved in a minimum of water was added. The suspension $\mathrm{pH}$ was slowly increased to a value just above the amine group $\mathrm{pK}_{\mathrm{b}}$ and the mixture was left on stirring for 30 minutes. During this time, unreacted aldehyde groups that were borne by the dextran chain reacted with amine groups present at the end of the peptide chains. Then, the $\mathrm{pH}$ was adjusted at 7.4 , the suspension was dialyzed for 30 minutes and lyophilized using the same membrane as for the dextran oxidation procedure. The obtained peptidedecorated fluorescent NPs were stored in the refrigerator.

\section{NPs characterization}

The NP size was measured with a NanoSight LM10 (Malvern Instruments, Malvern, UK) device employing Stokes Einstein equation to calculate size distribution. The size value was the average of 15 independent measurements, and the mean standard deviation was indicated. Zeta potentials were measured with the Zetasizer Nano ZS90 (Malvern Instruments). They were the average of ten independent measurements of 30 scans each. Freshly prepared NPs were used. Alternatively, NPs were kept at $4^{\circ} \mathrm{C}$ in lyophilized form for 6 months and then rehydrated for 30 minutes at the concentration of $0.1 \mathrm{mg} / \mathrm{mL}$ in buffered saline before measurements. To test aggregation, suspensions of NPs were kept at room temperature and the size distribution was analyzed at various time intervals. The fluorescence spectra were recorded on a SAFAS Xenius spectrofluorometer (SAFAS Monaco). Measurements on dry NP powders were performed using a $\mathrm{BaSO}_{4}$ integrating sphere. The procedure used for the measurement of photoluminescence quantum yields was identical to that described elsewhere. ${ }^{31}$

\section{In vitro studies}

In vitro studies were conducted on three different cell lines: OE19 (human esophageal carcinoma cells), BAR-T (human non-dysplastic/non-neoplastic BE cells), and Caco-2 (human colorectal adenocarcinoma cells). Cells were grown on a poly-L-lysine (Sigma-Aldrich) coating $(10 \mu \mathrm{g} / \mathrm{mL}$ in sterilized water) for 24 hours and then incubated with $100 \mu \mathrm{g} / \mathrm{mL}$ of NPs for 1.5 hours at $37^{\circ}$. Cells were washed and fixed with formaldehyde 4\% (Sigma-Aldrich). Total number of cells was visualized with the fluorescent nuclear dye 4',6-diamidino-2-phenylindole (Thermo Fisher Scientific) and coverslipped with Polyscience solution (Polysciences Europe GmbH, Eppelheim, Germany). Stained cells were examined with Leica TCS SP5 confocal microscope (Leica Microsystems, Wetzlar, Germany).

\section{Animal study approval}

Procedures involving animals and their care were conducted according to Italian law on the use of experimental animals (DL n. 116/92 art. 5), which acknowledges the European Directive 86/609 EU. The project proposal was approved by the Ethical Committee of University of Padua (Comitato Etico di Ateneo sulla Sperimentazione Animale-CEASA). In this pilot study, 17 male Sprague Dawley rats (Charles River, Lecco, Italy) were consecutively submitted to a surgical procedure to induce gastroesophageal reflux. The animals were kept under standard laboratory conditions and acclimatized for a week before the procedure. Water and standard chow food were given ad libitum before surgery. Water was permitted 2 hours after surgery, and rat chow was provided on the following day. Postoperatively, the animals were housed individually in a conventional cage. Thirteen male non-operated rats of the same age were used as control animals (Table 1).

\section{Anesthesia and surgical procedure to induce esophageal cancer}

Anesthesia was given using isoflurane (Forane ${ }^{\circledR}$, Abbott SpA, Campoverde, Milano, Italy) 3\% for induction and 1.5\% for 
Table I Number of animals used

\begin{tabular}{|c|c|c|}
\hline & $\begin{array}{l}\text { Non-operated } \\
\text { (control) }\end{array}$ & $\begin{array}{l}\text { Operated } \\
\text { ( } 80 \text { weeks ps) }\end{array}$ \\
\hline No injection & I & $2(\mathrm{EAC})$ \\
\hline NP-DCM (tp) ${ }^{\mathrm{a}}$ & 3 & 4 (I BE, 3 EAC) \\
\hline NP-DCM-ASYNYDA (tp) & 3 & $3(\mathrm{EAC})$ \\
\hline NP-DCM (24 hours) & 3 & $3(\mathrm{EAC})$ \\
\hline NP-DCM-ASYNYDA (24 hours) & 3 & 5 (2 BE, 3 EAC) \\
\hline Total & 13 & 17 \\
\hline
\end{tabular}

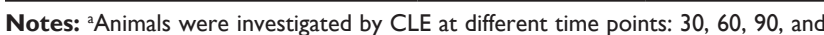
120 minutes after NP administration.

Abbreviations: $\mathrm{BE}$, Barrett's esophagus; CLE, confocal laser endomicroscopy; DCM, 4-(dicyanomethylene)-2-methyl-6-(4-dimethylaminostyryl)-4H-pyran; EAC, esophageal adenocarcinoma; NP, nanoparticle; ps, post-surgery; tp, time points.

maintenance, and oxygen $1 \mathrm{~L} / \mathrm{min}^{32}$ The animals were given

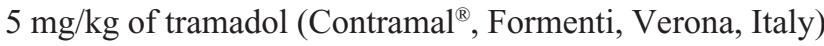
intraperitoneally immediately after the peritoneal incision. At the end of the surgical procedure, the animal was roused, maintaining $1 \mathrm{~L} / \mathrm{min}$ oxygen. The animals received $5 \mathrm{~mL}$ saline solution subcutaneously and intramuscular injections of tylosin $20 \mathrm{mg} / \mathrm{kg}$ (Depotyl-LA ${ }^{\circledR}$, Happyfarma, Vicenza, Italy) to prevent dehydration and surgical infections. None of the above-mentioned drugs are known as carcinogens. The operation was performed according to the microsurgical procedure previously described. ${ }^{23,24}$ Briefly, a $1.5 \mathrm{~cm}$ side-toside surgical esophagogastric-jejunal anastomosis was created between the first jejunal loop and the gastroesophageal junction, approximately $3 \mathrm{~cm}$ distal to Treitz's ligament, with accurate mucosa-to-mucosa opposition, allowing to jejunal and gastric contents to flow back into the esophagus. Animals were sacrificed 80 weeks post-surgery.

\section{NPs delivery}

NP-DCM and NP-DCM-ASYNYDA were diluted in filtered saline solution at the concentration of $5 \mathrm{mg} / \mathrm{mL}$ and administered iv in healthy and operated animals $(5 \mathrm{mg} / \mathrm{kg}$, three to five animals/condition). Rats were divided into two groups: group A was composed by animals which received via iv route NPs at time 0 and investigated by CLE at 30, 60,90 , and 120 minutes; group B was composed by animals which received via iv route NPs at time 0 and investigated by CLE after 24 hours.

\section{Murine CLE}

We used the "Cellvizio Dual Band" system integrated with Proflex Ultra Mini Z probes (Mauna Kea Technologies) and a flexible endoscope (MAS-GM, Olympus, Italia Srl, Milano, Italy) as previously described. ${ }^{25}$ Briefly, the tumor was first localized with the use of the endoscope under endotracheal intubation. The hand-held confocal probe, inserted in the operative channel of the endoscope, was positioned to achieve full contact with the tissue. Images from the whole tumor were collected. The whole distal rat esophagus was screened by carefully moving the probe across the surface and adapting the imaging plane depth. Post-processing imaging analysis was performed by the IC-viewer software. CLE was carried out on anesthetized non-operated and operated rats. CLE images were acquired at different time points, according to animal group.

\section{Histopathology}

The entire esophagus was removed and opened longitudinally to examine for evidence of gross morphological changes. First, evidence of reflux esophagitis, BE, and EAC was investigated macroscopically. Consecutively, gross specimens were fixed in 4\% neutral-buffered formalin for at least 24 hours, cut serially with $2-3 \mathrm{~mm}$ thick sections, and wholly embedded in paraffin. Tissue sections ( $4 \mu \mathrm{m}$ thick) were routinely stained with hematoxylin and eosin. Two experienced gastrointestinal pathologists reviewed all the slides in a blinded fashion. Every slide was evaluated for the following histopathological parameters: esophagitis (lamina propria inflammation, squamous epithelium hyperplasia/acanthosis/ spongiosis), BE (intestinal metaplasia), and EAC.

\section{Results and discussion NPs preparation}

Several steps were necessary for the preparation of NPs. In a first time, the controlled oxidation of dextran using periodate allowed the polysaccharide backbone to be preserved while some glucose rings were opened along the chain, thus generating reactive aldehyde groups, two per single opened glucose ring. These aldehyde groups may react with dodecylamine to form Schiff bases, resulting in hydrophilic dextran chain with hydrophobic side groups. This polymeric surfactant has a tendency to self-assemble and form NPs in water solution. ${ }^{33}$ This process was adapted to the preparation of polysaccharide-based fluorescent NPs. To do so, a microfluidic device was developed. During the production of fluorescent polysaccharide NPs, a few processes took place simultaneously. On the one hand, the formation of Schiff bases between oxidized dextran and dodecylamine occurred in the precipitating solution (B). Contemporarily in the microfluidic system, the DCM ethanol solution (A) was mixed with water solution, which generally causes nucleation and precipitation of the water-insoluble organic dye as nanocrystals. In this system, the growth of dye 
nanocrystals was modified by the presence of dodecylamine/ dextran conjugate. This led to the formation of fluorescent polysaccharide NPs.

In water, oxidized dextran can be reacted with molecules bearing a free amino group, to form biocompatible hydrogel coatings. ${ }^{34}$ The method is highly versatile and allows the binding of a variety of biologically-active molecules. In the present case, peptides were grafted on the fluorescent polysaccharide NPs using this Schiff base reaction. To do so, a large number of peptides containing the ASYNYDA sequence were designed with different peptide spacer sequences in order to optimize the display/exposition of the peptide on the NP surface. The final peptide used in this study was Ac-ASYNYDAGGGSK(Ahx)- $\mathrm{NH}_{2}$ equipped with an unnatural residue, aminohexanoic acid (Ahx) branched at the side chain of the $\mathrm{C}$-terminal lysine residue to allow covalent binding with the aldehyde groups of the modified dextran. The peptide-decorated NPs (NP-DCM-ASYNYDA), as well as plain NPs (NP-DCM) used for the sake of comparison, were lyophilized.

\section{NPs characterization}

First of all, the peptide-conjugated NPs were analyzed by MALDI-TOF/TOF MS. The mass spectrum showed a signal corresponding exactly to the peptide molecular weight. Several species due to the fragmentation of dextran were also detected. They differed from each other by 162 Da, which corresponds to the hexosyl unit (Figure 3A). Furthermore, NP-DCM-ASYNYDA NPs were treated with $\mathrm{HCl}$, in order to hydrolyze the imine bond responsible for the covalent binding of peptides. The NPs were then removed by dialysis, and the solution was analyzed by MS. MS analysis clearly disclosed the presence of the ASYNYDA peptide in solution as a result of $\mathrm{HCl}$ hydrolysis (Figure 3B). In the absence of $\mathrm{HCl}$ hydrolysis, no peptide was detected in solution after dialysis. These experiments unambiguously showed that peptides were covalently bound to dextran.

In parallel, the physical characteristics of NPs were determined at various stages of their preparation. Measurements by NanoSight are particularly well adapted to fluorescent NPs. They showed that native fluorescent polysaccharide NPs had a hydrodynamic diameter of approximately $185 \pm 26$ $\mathrm{nm}$, with quite a wide size distribution (Figure 4A). After freeze drying and rehydration, the NP size was $148 \pm 3 \mathrm{~nm}$, and the distribution was much narrow, showing that the NPs markedly shrunk during this process (Figure 4C). Native peptide-decorated NPs (NP-DCM-ASYNYDA), which were
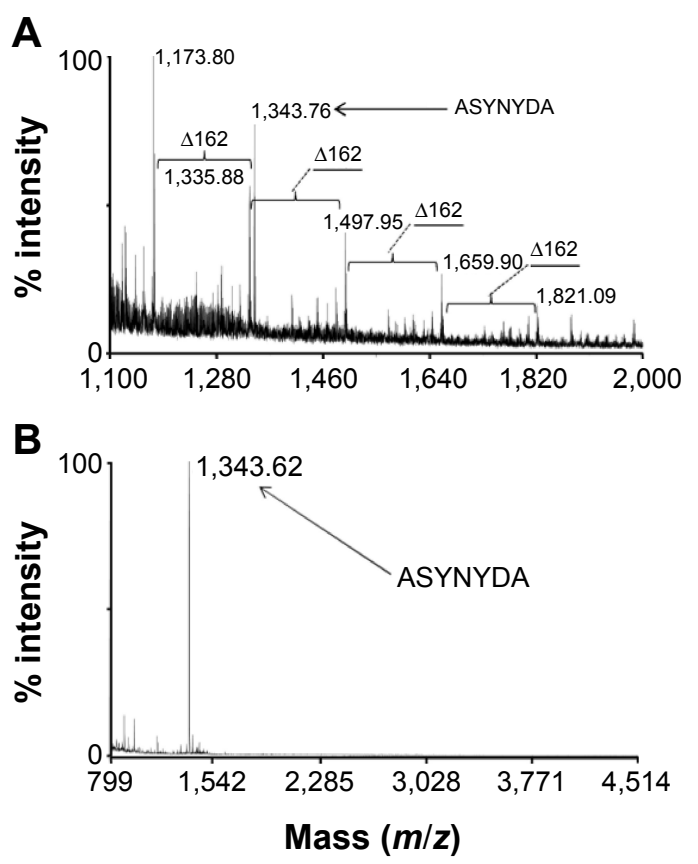

Figure 3 Characterization of NPs-DCM-ASYNYDA nanoparticles by MALDI-TOF/ TOF analysis.

Notes: (A) MS spectrum of NP-DCM-ASYNYDA. The value $\mathrm{m} / \mathrm{z}, \mathrm{I}, 343.76$, is consistent with the sequence of the peptide Ac-ASYNYDAGGGSK(Ahx)- $\mathrm{NH}_{2}$ (theoretical value $=1,343.69 \mathrm{~m} / \mathrm{z}$ ) grafted to the NPs. The signals derived from the dextran coating fragmentation differed by $162 \mathrm{Da}$ as indicated. (B) MS analysis of dialyzed solution of NP-DCM-ASYNYDA following treatment with $\mathrm{HCl}$. The main molecular species present in solution is the peptide $(1,343.62 \mathrm{~m} / \mathrm{z})$ removed by hydrolysis.

Abbreviations: DCM, 4-(dicyanomethylene)-2-methyl-6-(4-dimethylaminostyryl)$4 \mathrm{H}$-pyran; MS, mass spectrometry; NPs, nanoparticles; MALDI-TOF/TOF, matrixassisted laser desorption/ionization time-of-flight.

made using lyophilized polysaccharide NPs, had a diameter of $152 \pm 5 \mathrm{~nm}$ (Figure 4B). Addition of peptides therefore, induced a very small change in size. Subsequent freezedrying process induced little modification of the diameter

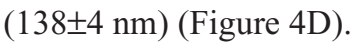

The zeta $(\zeta)$ surface potentials were measured on rehydrated NPs. A small negative $\zeta$-value of $-3.24 \pm 0.69 \mathrm{mV}$ was found for NP-DCM, and this value was even lower for NP-DCM-ASYNYDA $(-0.97 \pm 0.31 \mathrm{mV})$. This observation showed that the binding of peptides modified the $\zeta$ potential value of the NP surface. Despite these low $\zeta$-values, agglomeration of both NP-DCM and NP-DCM-ASYNYDA in saline buffer was only detected after 96 hours. It was also checked that NPs stored in lyophilized form for 1 year before rehydration exhibited no change in physicochemical parameters.

According to the synthesis conditions, the concentration of peptide with respect to NP-DCM was $0.18 \%$ in weight. Taking into account the size of the NPs and assuming that all peptides were bound to the NPs, the estimated average was 10 peptide molecules per NP, and at least one proportion of the peptides was situated near the NP surface. Assuming that all dye was 

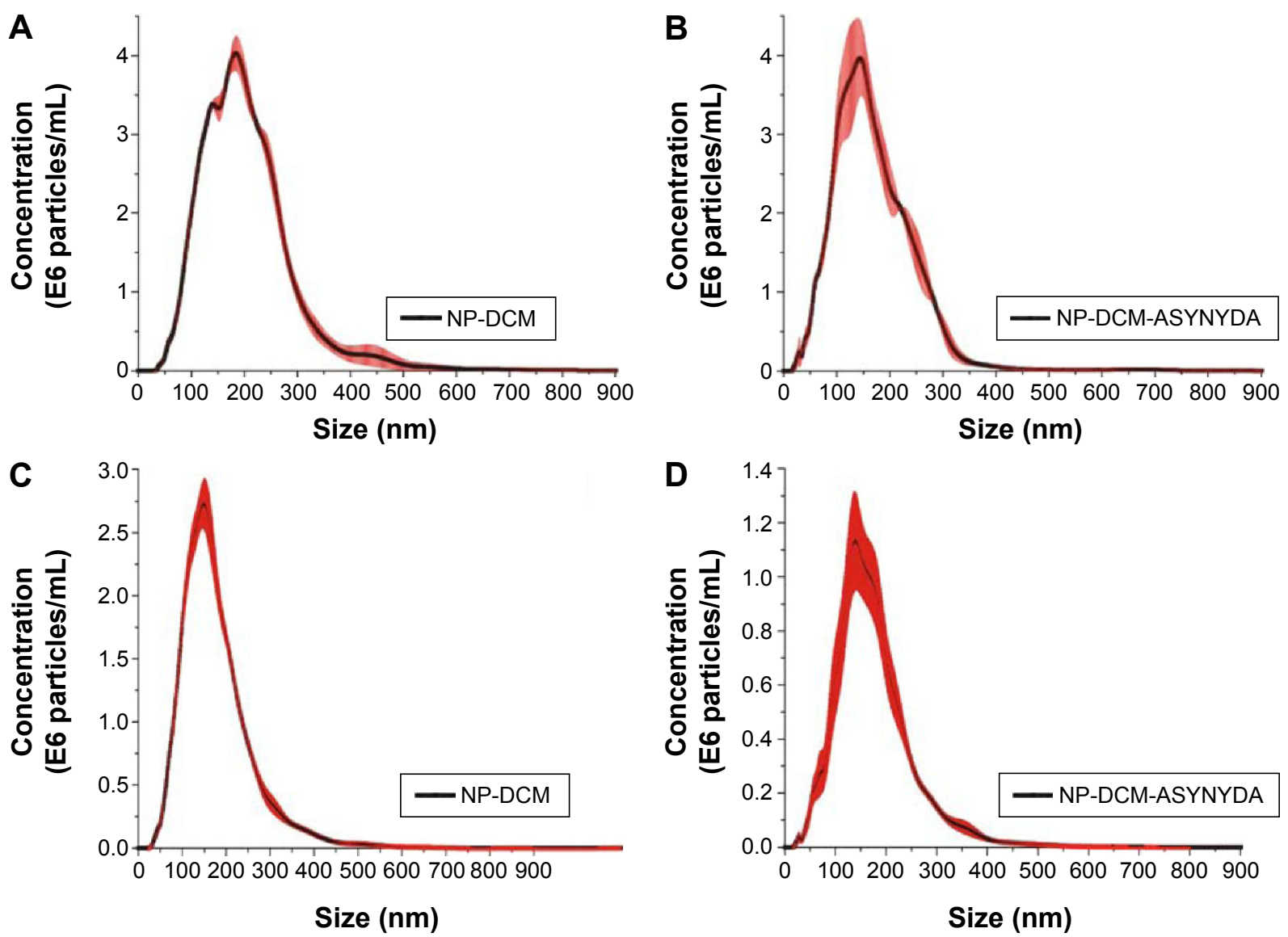

Figure 4 Size distribution of fluorescent polysaccharide NPs.

Notes: (A and B) Size distribution of NP-DCM and NP-DCM-ASYNYDA before lyophilization; (C and D) size distribution of NP-DCM and NP-DCM-ASYNYDA after rehydration. (NanoSight measurements).

Abbreviations: DCM, 4-(dicyanomethylene)-2-methyl-6-(4-dimethylaminostyryl)-4H-pyran; NP, nanoparticle.

incorporated in NPs, the average DCM dye loading was calculated to be approximately $1.2 \%$ in weight in NP-DCM.

Finally, the optical properties were examined. NP-DCM was rehydrated for 30 minutes in saline buffer. The obtained suspension was strongly light scattering, preventing the accurate measurement of the absorption spectrum. In contrast, good fluorescence spectra were recorded. NP-DCM exhibited a broad excitation spectrum centered at around $495 \mathrm{~nm}$ and an emission spectrum peaking at around $622 \mathrm{~nm}$. This emission value is close to that reported for DCM in ethanol. ${ }^{35}$ Photoluminescence of dry NP-DCM powders was also studied. By excitation at $488 \mathrm{~nm}$, the solid-state emission spectrum was close to that recorded in solution (Figure S1). This could indicate that the dye was protected from the aqueous environment. However, the broadness of the excitation and emission spectra that partly overlapped, the presence of shoulders, and the variations of the emission spectra shape with varying the excitation wavelength indicated that the fluorophore molecules experienced various environments. The low photoluminescence quantum yield of $3 \%$ suggested that at least a proportion of the dye was in the solid state. ${ }^{36}$

\section{NP-DCM-ASYNYDA specifically binds to esophageal cancer cells}

In vitro studies were performed to evaluate NPs' ability to recognize esophageal cancer cells and their cellular localization. To this purpose, human Caucasian esophageal carcinoma cell line (OE19), human non-dysplastic/non-neoplastic BE cell line (BAR-T), and human colorectal adenocarcinoma cell line (Caco-2) were incubated with NPs and fluorescence signal was analyzed by confocal microscopy. As expected, results showed a strong fluorescent signal in OE19 cells after NP-DCM-ASYNYDA incubation. Weak fluorescent immunoreactivity was observed in OE19 cells incubated with non-decorated NPs (NP-DCM). Only a very weak signal was observed in BAR-T cells with both NPs formulations and, as expected, no signal was observed in colorectal cancer cell line Caco-2 (Figure S2). Similar to previous studies, our data supported ASYNYDA binding specificity toward 
esophageal cancer cells. Notably, immunoreactivity was observed on the cell plasma membrane, according to the results of Sturm et al. ${ }^{19,20}$

\section{NPs reach esophageal cancerous tissue but not healthy and BE tissue}

As we reported in a previous study, ${ }^{25}$ and as already described by other researchers, ${ }^{37}$ gastroesophageal reflux induced by surgical esophagogastric-jejunal anastomosis promotes in our experimental animal model a spontaneous form of EAC in BE.

The endoscopic procedure was performed in healthy and operated animals. Endoscopic examination of control nonoperated rats did not reveal any abnormal findings, whereas we observed the endoscopic appearance of the different types of mucosa (mild esophagitis, BE, and EAC in BEEAC mucosa) in operated rats. As expected, the normal rat esophageal mucosa showed a smooth and white epithelium with a distinct vascular pattern. On the contrary, some of the operated rats displayed a mucosa characteristic of intestinal metaplasia. BE mucosa was identified from the appearance of any of the following mucosa patterns: elevated plaques/ ridges, deep grooves, or thin linear folds with a reduced vascular pattern. At endoscopic level, operated rats mainly developed esophageal cancer mucosa characterized by irregular shaped mass lesions with ulcerations and uneven, rough, friable mucosa. Histological analyses were consistent with endoscopic findings. After hematoxylin and eosin staining, microscopy imaging demonstrated that the red mucosa had the histological features of intestinal metaplasia and EAC, respectively, confirming the endoscopic findings (Figure 5).

Murine endoscopy and CLE were performed after tail vein injection of NP-DCM or NP-DCM-ASYNYDA at time 0 and analyzed every 30 minutes up to 2 hours (group A) and after 24 hours (group B) (Table 1). No difference of the uptake of NPs between the groups was observed
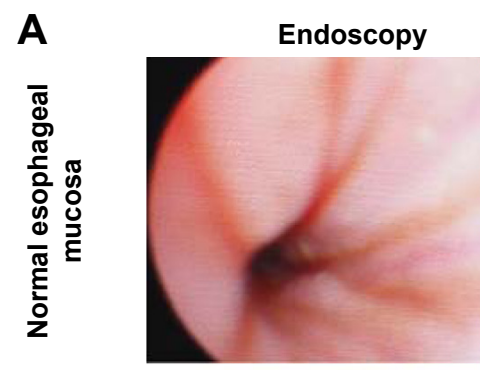

B
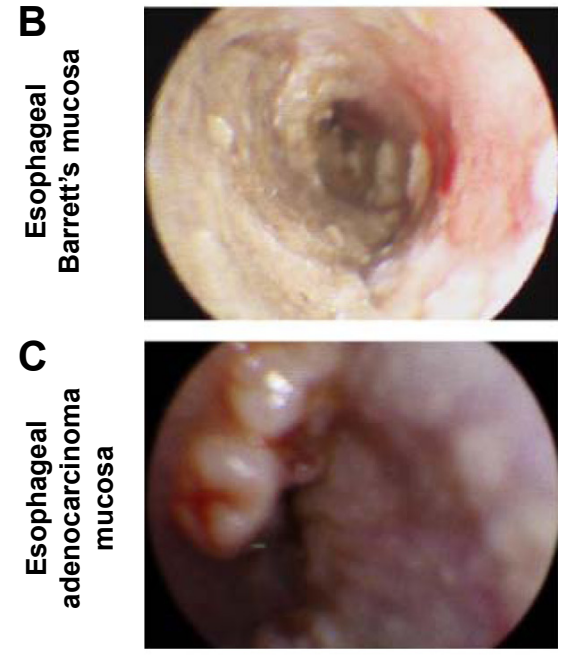
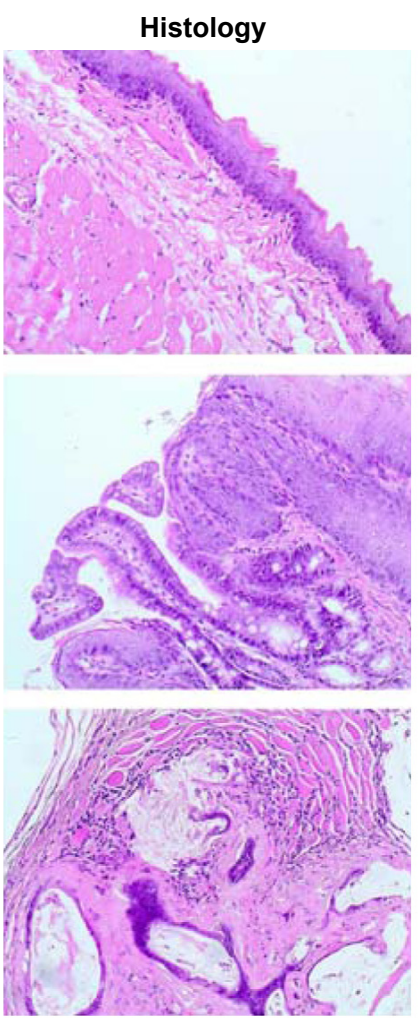

CLE before treatment
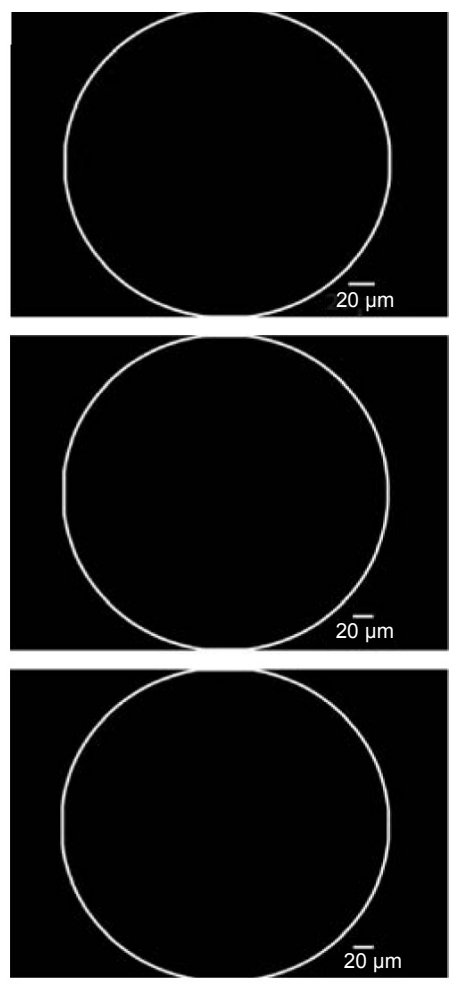

Figure 5 Endoscopic view, histology, and confocal imaging of rat esophageal mucosa before NP administration.

Notes: (A) Endoscopic view of normal mucosa which shows a smooth and white epithelium with a distinct vascular pattern. Histology of normal esophageal mucosa: normal squamous epithelium layering on thin lamina propria and chorion devoid of pathologic changes (ie, inflammation). H\&E, original magnification I00X. CLE imaging before NP administration of a normal rat esophageal mucosa did not show any fluorescent signal. (B) Endoscopic view of esophageal Barrett's mucosa, which shows elevated plaques/ ridges, deep grooves, and thin linear folds with a reduced vascular pattern. Histology of esophageal Barrett's mucosa with evident intestinal metaplasia of intervening gland. H\&E, original magnification 100×. CLE imaging before NP administration of a rat affected by Barrett's esophagus did not show any fluorescent signal. (C) Endoscopic view of esophageal adenocarcinoma mucosa, which shows an irregular shaped mass lesions with ulcerations and uneven, rough, friable mucosa. Histology of mucinous adenocarcinoma, with invasion of muscularis propria, irregularly shaped and dilated glands with large amount of intraluminal mucin. H\&E, original magnification I00X. CLE imaging before NP administration of a rat affected by esophageal adenocarcinoma did not show any fluorescent signal.

Abbreviations: CLE, confocal laser endomicroscopy; H\&E, hematoxylin and eosin; NP, nanoparticle. 
between totally normal esophageal mucosa and mucosa esophagitis without BE or simple BE. Fluorescence signal was detected in correspondence with tumors grown in the distal part of the esophagus, at level of the anastomotic site (Figure 6). In group A animals, a weak and diffuse fluorescence signal appeared 30 minutes post-NP administration and became stronger after 2 hours (last time point analyzed for rats belonging to group A). Signal intensity was more robust and more localized in animals injected 24 hours before endoscopy (group B) (Video S1 in operated rats upon iv administration of NP-DCM, Video S2 in operated rats upon iv administration of NP-DCM-ASYNYDA).
The fluorescence signal was detected where growing tumors were observed, without following any specific anatomical architecture, suggesting that NPs bound to esophageal tumor cells and not to non-cancer cells or any other cell type, such as blood vessel endothelial cells. Both NP-DCM and NPDCM-ASYNYDA had the ability to cross the endothelium of the tumor microvasculature and to be retained by cancer tissue, suggesting that the NPs reached tumor cells possibly by enhanced permeability and retention effect, but proving the incorporation process would require further investigations. Interestingly, fluorescence intensity in EAC tissue was stronger in animals injected with NP-DCM-ASYNYDA

A

NP-DCM (30 minutes to 24 hours)
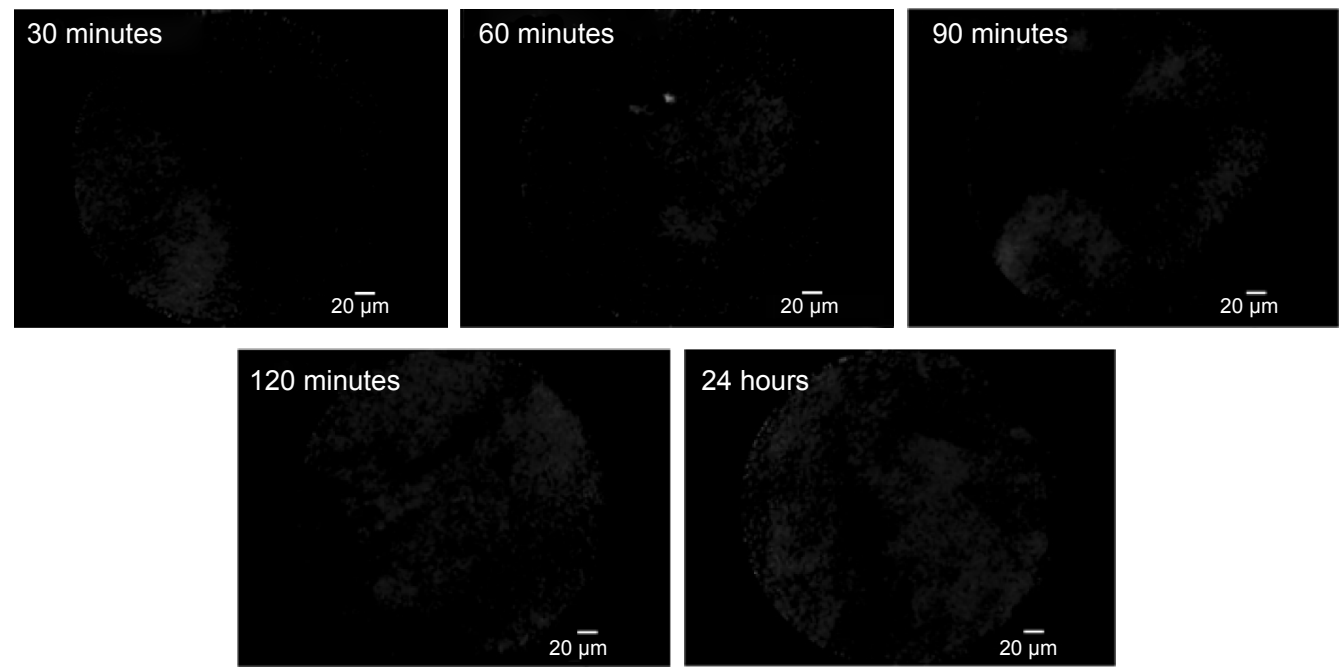

B

NP-DCM-ASYNYDA (30 minutes to 24 hours)
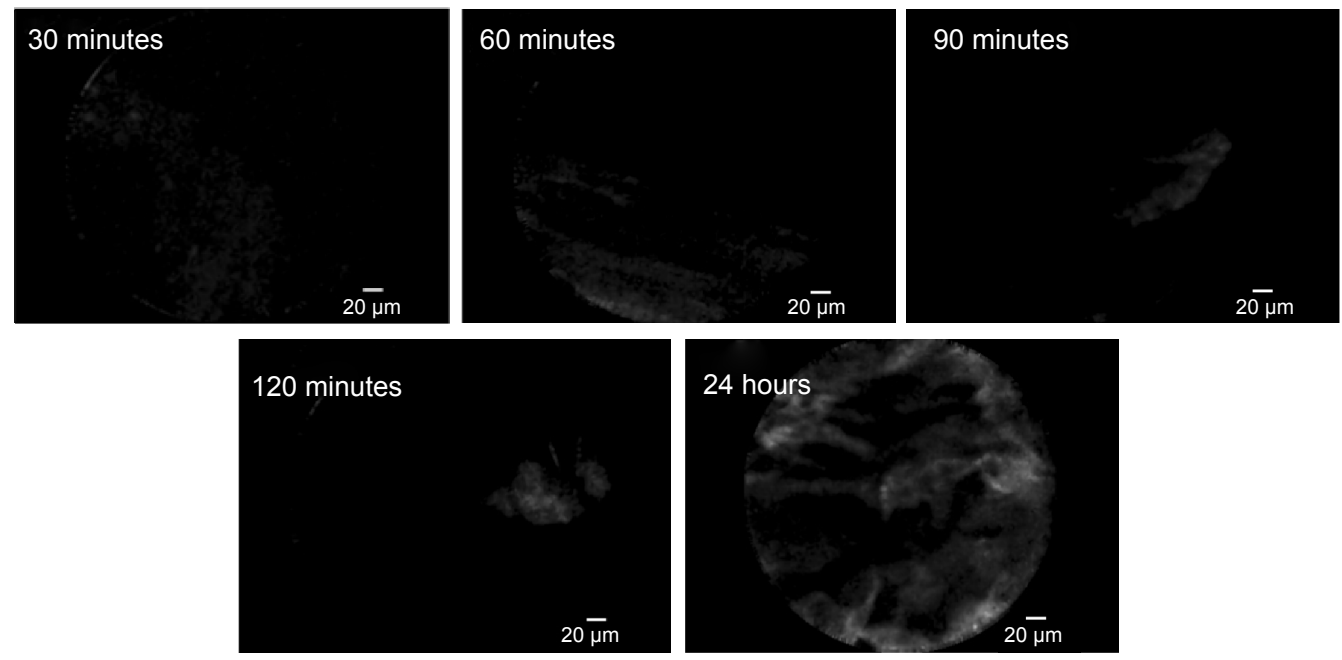

Figure 6 Confocal imaging of rat esophageal adenocarcinoma upon intravenous administration of NPs.

Notes: (A) CLE images upon intravenous administration of NP-DCM were acquired after 30, 60, 90, 120 minutes, and 24 hours. Their weak and diffuse fluorescent signal became noticeable after 30 minutes and was stronger and more localized after 24 hours. (B) CLE images upon intravenous administration of NP-DCM-ASYNYDA were acquired after 30, 60, 90, 120 minutes, and 24 hours. The fluorescent signal was particularly strong and specific after 24 hours.

Abbreviations: DCM, 4-(dicyanomethylene)-2-methyl-6-(4-dimethylaminostyryl)-4H-pyran; CLE, confocal laser endomicroscopy; NP, nanoparticle. 
compared with animals injected with NP-DCM in both groups, either in the short (30 minutes to 2 hours) and in the long ( 24 hours) period. This observation confirmed our in vitro results and the specificity of the heptapeptide ASYNYDA for high-grade dysplasia and EAC cells that has been reported in the literature. ${ }^{20}$

\section{Conclusion}

Current diagnostic strategies and treatments for EAC need to be improved in order to offer an early diagnosis and novel treatment alternatives to increase the present poor response rates. In this preliminary study, the non-invasive approach CLE was used to investigate the ability of fluorescent NPs to recognize murine esophageal cancer cells. Our NP design and synthesis strategy allowed us to obtain NPs with distinct advantages. NPs were totally organic, with very low toxicity. The coating made of a modified polysaccharide provided stability, biocompatibility and furtivity toward the immune system. The modified polysaccharide made these NPs bioavailable, offering new opportunities for further surface engineering. Interestingly, our work showed that NP-DCM grafted with ASYNYDA peptide had high specificity for human and rat esophageal cancer cells. The proof of concept of our approach is thus obtained.

Our NPs were designed with diagnostic purpose to identify dysplastic or early esophageal neoplastic lesions. Currently, the surveillance endoscopy is the primary management approach for BE with random four-quadrant biopsies taken every $1-2 \mathrm{~cm}$ along the BE segment, at designated intervals. However, standard protocol biopsies have been associated with a miss rate of up to $57 \%$ for dysplastic/ neoplastic lesions in patients with BE. ${ }^{38}$ This is largely due to the fact that dysplasia or neoplasia may be focal, flat, and endoscopically indistinguishable from non-neoplastic epithelium on routine white-light endoscopy. Since the first visible neoplastic changes in epithelial cancers occur at a cellular level, CLE may allow for earlier diagnosis and treatment: it could dramatically improve the imaging capabilities of flexible endoscopy by its ability to collect "optical biopsies" of endoluminal surface, thereby decreasing the number of biopsies required to diagnose dysplasia or neoplasia in daily routine.

Of course, further studies are needed to answer some technical questions. First, a more efficient fluorescent dye must be developed and its spectroscopic behavior fully studied. The preparation of NPs must be optimized. Although no toxic effect was observed, a quantitative study concerning kinetic properties, biodistribution, metabolism, and elimination should be further undertaken.
Finally, the same type of NPs can incorporate bioactive compounds to develop targeted therapeutic or theranostic applications. NPs grafted with molecules or peptides able to recognize known tumor markers (eg, NPs grafted with anti-tumoral compounds) could be generated as a diagnostic tool for many other types of cancer. These numerous and exciting possibilities encourage further developments of the platform and additional studies that are required before translation into clinical practice.

\section{Acknowledgments}

The research was funded by EuroNanoMed (JTC-2011, FONDIAG project), AIRC (Italian Association for Cancer Research, project no 10761), and Ricerca Corrente IOV (2014). We are grateful to L Vidotto for technical contribution to histology. The funding agency had no role in the design and conduct of the study.

\section{Disclosure}

The authors report no conflicts of interest in this work.

\section{References}

1. Pohl H, Welch HG. The role of overdiagnosis and reclassification in the marked increase of esophageal adenocarcinoma incidence. J Natl Cancer Inst. 2005;97(2):142-146.

2. Siegel R, Ma J, Zou Z, Jemal A. Cancer statistics, 2014. CA Cancer J Clin. 2014;64(1):9-29.

3. Bennett M, Mashimo H. Molecular markers and imaging tools to identify malignant potential in Barrett's esophagus. World J Gastrointest Pathophysiol. 2014;5(4):438-449.

4. Phillips WA, Lord RV, Nancarrow DJ, Watson DI, Whiteman DC. Barrett's esophagus. J Gastroenterol Hepatol. 2011;26(4):639-648.

5. Dulai GS, Guha S, Kahn KL, Gornbein J, Weinstein WM. Preoperative prevalence of Barrett's esophagus in esophageal adenocarcinoma: a systematic review. Gastroenterology. 2002;122(1):26-33.

6. Zhang WJ, Sui YX, Budha A, et al. Affinity peptide developed by phage display selection for targeting gastric cancer. World J Gastroenterol. 2012; 18(17):2053-2060.

7. Xu D, Wu F, Chen Y, Wei L, Yuan W. pH-sensitive degradable nanoparticles for highly efficient intracellular delivery of exogenous protein. Int J Nanomedicine. 2013;8(1):3405-3414.

8. Maeda H. Tumor-selective delivery of macromolecular drugs via the EPR effect: background and future prospects. Bioconjug Chem. 2010;21(5): 797-802.

9. Bigini P, Previdi S, Casarin E, et al. In vivo fate of avidin-nucleic acid nanoassemblies as multifunctional diagnostic tools. ACS Nano. 2014;8(1): 175-187.

10. Moghimi SM, Hunter AC, Murray JC. Long-circulating and targetspecific nanoparticles: theory to practice. Pharmacol Rev. 2001;53(2): 283-318.

11. Goetz M, Wang TD. Molecular imaging in gastrointestinal endoscopy. Gastroenterology. 2010;138(3):828-833.

12. Johnson EA, De Lee R, Agni R, Pfau P, Reichelderfer M, Gopal DV. Probe-based confocal laser endomicroscopy to guide real-time endoscopic therapy in Barrett's esophagus with dysplasia. Case Rep Gastroenterol. 2012;6(2):285-292.

13. Goetz M, Kiesslich R. Confocal endomicroscopy: in vivo diagnosis of neoplastic lesions of the gastrointestinal tract. Anticancer Res. 2008;28(1B): 353-360. 
14. Bertani H, Pigò F, Dabizzi E, et al. Advances in endoscopic visualization of Barrett's esophagus: the role of confocal laser endomicroscopy. Gastroentero Res Pract. 2012;2012:493961.

15. Buda A, Facchin S, Dassie E, et al. Detection of a fluorescent-labeled avidin-nucleic acid nanoassembly by confocal laser endomicroscopy in the microvasculature of chronically inflamed intestinal mucosa. Int J Nanomedicine. 2015;10:399-408.

16. Buda A, Hatem G, Neumann H, et al. Confocal laser endomicroscopy for prediction of disease relapse in ulcerative colitis: a pilot study. J Crohns Colitis. 2014;8(4):304-311.

17. Wang R, Xiao R, Zeng Z, Xu L, Wang J. Application of poly(ethylene glycol)-distearoylphosphatidylethanolamine (PEG-DSPE) block copolymers and their derivatives as nanomaterials in drug delivery. Int J Nanomedicine. 2012;7:4185-4198.

18. Shin SJ, Beech JR, Kelly KA. Targeted nanoparticles in imaging: paving the way for personalized medicine in the battle against cancer. Integr Biol (Camb). 2013;5(1):29-42.

19. Sturm MB, Joshi BP, Lu S, et al. Targeted imaging of esophageal neoplasia with a fluorescently labeled peptide: first-in-human results. Sci Transl Med. 2013;5(184):184ra61.

20. Sturm MB, Piraka C, Elmunzer BJ, et al. In vivo molecular imaging of Barrett's esophagus with confocal laser endomicroscopy. Gastroenterology. 2013;145(1):56-58

21. Raggi M, Langer R, Feith M, Friess H, Schauer M, Theisen J. Successful evaluation of a new animal model using mice for esophageal adenocarcinoma. Langenbecks Arch Surg. 2010;395(4):347-350.

22. Garman KS, Orlando RC, Chen X. Review: experimental models for Barrett's esophagus and esophageal adenocarcinoma. Am J Physiol Gastrointest Liver Physiol. 2012;302(11):G1231-G1243.

23. Ingravallo G, Dall'Olmo L, Segat D, et al. CDX2 hox gene product in a rat model of esophageal cancer. J Exp Clin Cancer Res. 2009;28:108: $1-6$.

24. Oh DS, DeMeester SR, Dunst CM, et al. Validation of a rodent model of Barrett's esophagus using quantitative gene expression profiling. Surg Endosc. 2009;23(6):1346-1352.

25. Realdon S, Dassie E, Fassan M, et al. In vivo molecular imaging of HER2 expression in a rat model of Barrett's esophagus adenocarcinoma. Dis Esophagus. 2015;28(4):394-403.

26. Dall'Olmo L, Fassan M, Dassie E, et al. Role of proton pump inhibitor on esophageal carcinogenesis and pancreatic acinar cell metaplasia development: an experimental in vivo study. PLoS One. 2014; 9(11):e112862.
27. Muangsiri W, Kirsch LE. The protein-binding and drug release properties of macromolecular conjugates containing daptomycin and dextran. Int J Pharm. 2006;315(1-2):30-43.

28. Fuentes M, Segura RL, Abian O, et al. Determination of protein-protein interactions through aldehyde-dextran intermolecular cross-linking. Proteomics. 2004;4(9):2602-2607.

29. Field GB, Noble RL. Solid phase peptide synthesis utilizing 9-fluorenylmethoxycarbonyl amino acids. Int J Pept Protein Res. 1990; 35(3):161-214.

30. Carpino LA, Henklein P, Foxman BM, et al. The solid state and solution structure of HAPyU. J Org Chem. 2001;66(15):5245-5247.

31. Khemakhem K, Soulié M, Brousses R, Ammar H, Abid S, Fery-Forgues S. Small iminocoumarin derivatives as red emitters: from biological imaging to highly photoluminescent non-doped micro- and nanofibres. Chem Eur J. 2015;21(21):7927-7937.

32. Lu S, Lowe AW, Triadafilopoulos G, et al. Endoscopic evaluation of esophago-gastro-jejunostomy in rat model of Barrett's esophagus. Dis Esophagus. 2009;22(4):323-330.

33. Ciach T, Wasiak I. Process for the preparation of polysaccharide nanoparticles. Patent WO 2013/137755 A1.

34. Lisman A, Butruk B, Wasiak I, Ciach T. Dextran/Albumin hydrogel sealant for Dacron (R) vascular prosthesis. J Biomater Appl. 2014;28(9): 1386-1396.

35. Rurack K, Spieles M. Fluorescence quantum yields of a series of red and near-infrared dyes emitting at 600-1,000 nm. Anal Chem. 2011;83(4): $1232-1242$.

36. Yan D, Fan G, Guan Y, Meng Q, Li C, Wang J. Tuning solid-state blue and red luminescence by the formation of solvate crystals. Phys Chem Chem Phys. 2013;15(45):19845-19852.

37. Li Y, Gobin AM, Dryden GW, et al. Infrared light-absorbing gold/gold sulfide nanoparticles induce cell death in esophageal adenocarcinoma. Int J Nanomedicine. 2013;8(1):2153-2161.

38. Vieth M, Ell C, Gossner L, May A, Stolte M. Histological analysis of endoscopic resection specimens from 326 patients with Barrett's esophagus and early neoplasia. Endoscopy. 2004;36(9):776-781. 


\section{Supplementary materials}

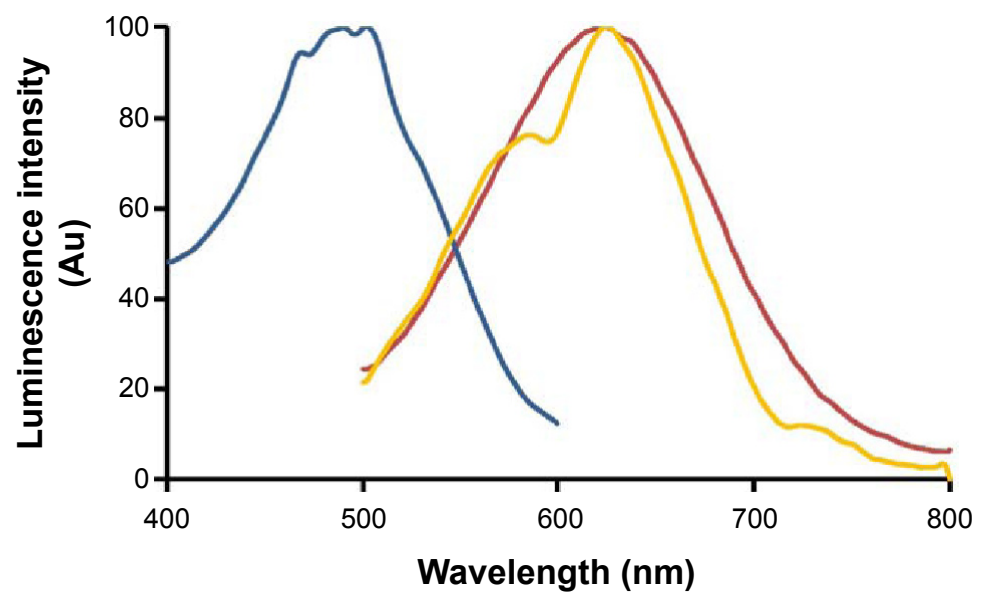

Figure SI Fluorescence excitation (blue line) and emission (red line) spectra of NP-DCM nanoparticles in saline buffer, and solid-state emission spectrum of the dry powder (orange line).

Notes: For emission spectra, $\lambda_{\text {ex }}=488 \mathrm{~nm}$. For excitation spectra, $\lambda_{\mathrm{em}}=620 \mathrm{~nm}$.

Abbreviations: DCM, 4-(dicyanomethylene)-2-methyl-6-(4-dimethylaminostyryl)-4H-pyran; NP, nanoparticle.
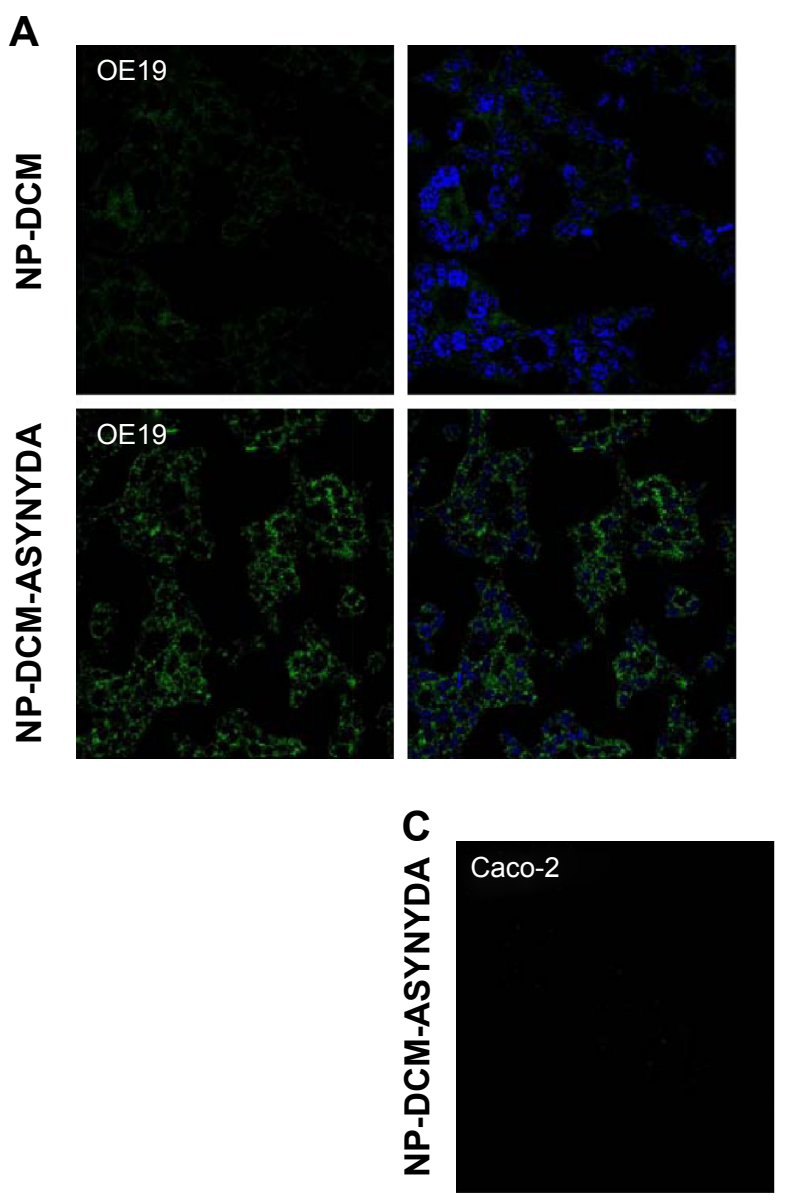
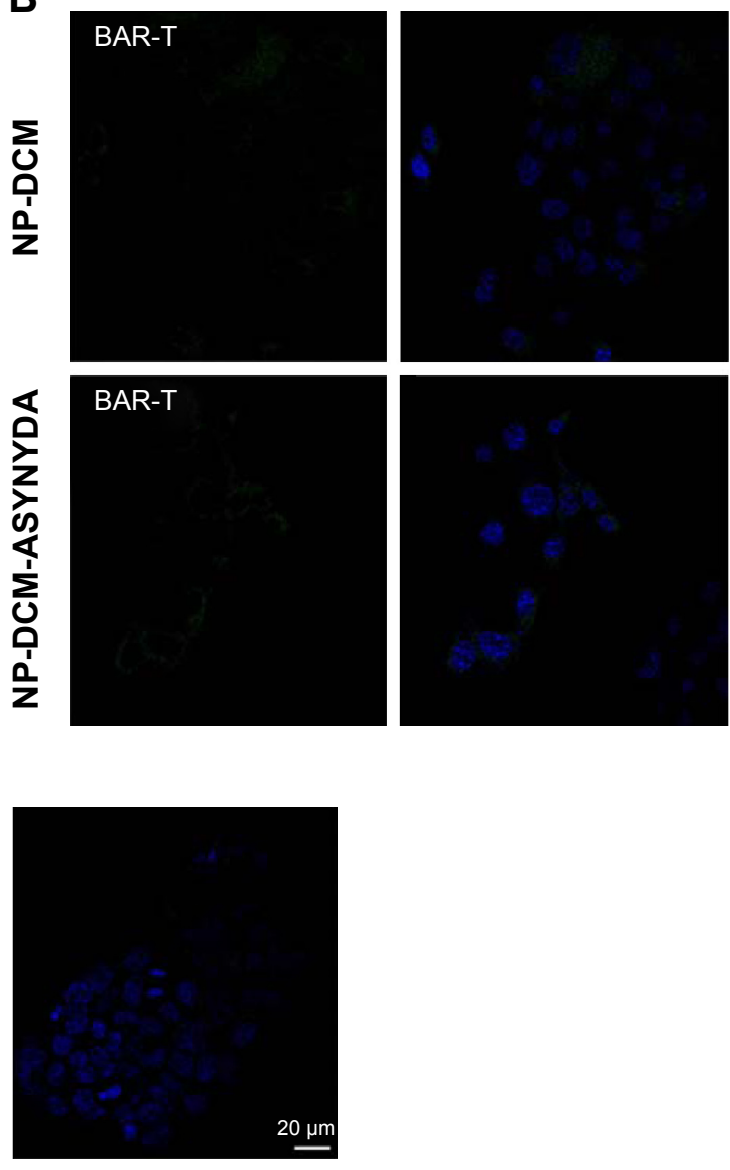

Figure S2 NP-DCM-ASYNYDA binds to human esophageal carcinoma cells.

Notes: (A) NP-DCM fluorescent signal was observed in OEI9 cells, but it was qualitatively less robust compared with the fluorescent signal given by NP-DCM-ASYNYDA. (B) A very weak fluorescent signal was observed in BAR-T cells with both NPs formulations. (C) NP-DCM-ASYNYDA did not show fluorescent signal in Caco-2 cells. Abbreviations: DCM, 4-(dicyanomethylene)-2-methyl-6-(4-dimethylaminostyryl)-4H-pyran; NP, nanoparticle. 
Video SI CLE upon intravenous administration of NP-DCM acquired after 24 hours.

Abbreviations: CLE, confocal laser endomicroscopy; DCM, 4-(dicyanomethylene)-2-methyl-6-(4-dimethylaminostyryl)-4H-pyran; NP, nanoparticles.

Video 52 CLE upon intravenous administration of NP-DCM-ASYNYDA acquired after 24 hours.

Abbreviations: CLE, confocal laser endomicroscopy; DCM, 4-(dicyanomethylene)-2-methyl-6-(4-dimethylaminostyryl)-4H-pyran; NP, nanoparticles.

\section{Publish your work in this journal}

The International Journal of Nanomedicine is an international, peerreviewed journal focusing on the application of nanotechnology in diagnostics, therapeutics, and drug delivery systems throughout the biomedical field. This journal is indexed on PubMed Central, MedLine, CAS, SciSearch $®$, Current Contents $® /$ Clinical Medicine,
Journal Citation Reports/Science Edition, EMBase, Scopus and the Elsevier Bibliographic databases. The manuscript management system is completely online and includes a very quick and fair peer-review system, which is all easy to use. Visit http://www.dovepress.com/ testimonials.php to read real quotes from published authors.

Submit your manuscript here: http://www.dovepress.com/international-journal-of-nanomedicine-journal 